\title{
Tendinitis cálcica del manguito rotador y su lavado por ultrasonido
}

\author{
Stefan Guiloff $P^{1}$, Juan Pablo Niedmann $E^{2}$, Esteban Hebel $N^{1}$, Fabián Villacres $C^{1}$.
}

1. Residente de Radiología. Facultad de Medicina, Universidad del Desarrollo. Clínica Alemana. Santiago, Chile.

2. Médico Radiólogo. Clínica Alemana de Santiago. Santiago, Chile.

\section{Calcific tendinitis of the rotator cuff and its lavage using ultrasound}

\begin{abstract}
Purpose: The objective of this study is to describe the technique of lavage and aspiration of calcifications under ultrasound in patients affected by this entity and to evaluate their results in the short term. Experimental design: Retrospective, descriptive study, with approval from the Ethics Committee of the Institution. Material and Methods: We performed a retrospective search of patients who underwent lavage as well as shoulder aspiration under ultrasound for calcific tendinitis at the Clínica Alemana in Santiago. 94 patients were identified between the years 2011 and 2015, of which 23 were excluded due to incomplete data. The electronic clinical record was reviewed and the data was recorded in RedCap v6 and statistical analyzes were performed using StataSE v12. Results: The sample consisted of 71 patients, the median age being 50.1 years (IQR: 41.6-57.7) and $45.1 \%$ were women. $70.4 \%$ affected the right shoulder. The mean evolution of the symptoms was 5 months (IQR: 2-24 months). $22.5 \%$ received one or more previous infiltrations and $47.9 \%$ attended kinesiotherapy. The calcifications corresponded mainly to type 1 according to the Gartner and Heyer classification (73.3\%), with a median size of $14 \mathrm{~mm}$ on their major axis (IQR: 10-18 mm). They affected one tendon in $74.7 \%$, two tendons in $18.3 \%$ and three tendons in $7.0 \%$, the supraspinatus being the tendon most frequently affected (90.1\%). $85.9 \%$ presented complete or significant improvement of the symptoms, only $8.5 \%$ requiring surgical resolution with arthroscopy. The final improvement had no significant association with the Gartner and Heyer type $(p=0.3)$, size $(p=$ $0.16)$, or with the evolution time of the symptoms $(p=0.7)$. Conclusions: Patients with calcific tendinitis who underwent lavage and aspiration under ultrasound had, in most cases, a significant or total resolution of the symptoms at two months follow-up, with only a minor percentage requiring arthroscopy. It was not possible to identify predictors of poor evolution for this procedure. Further studies are needed to determine its advantages over medical treatment.
\end{abstract}

Key words: Tendinitis, calcific tendinitis, Rotator cuff, Ultrasonic lavage.

Resumen. Propósito: El objetivo de este estudio es describir la técnica de lavado y aspirado de calcificaciones bajo ultrasonido en pacientes afectados por esta entidad y evaluar sus resultados a corto plazo. Diseño experimental: Estudio retrospectivo, descriptivo, con aprobación del Comité de Ética de la Institución. Material y Métodos: Se realizó una búsqueda retrospectiva de los pacientes sometidos a lavado más aspirado de hombro bajo ultrasonido por tendinitis cálcica en Clínica Alemana de Santiago. Se identificaron 94 pacientes entre los años 2011 y 2015, de los cuales 23 fueron excluidos por presentar datos incompletos. Se revisó la ficha clínica electrónica y los datos fueron registrados en RedCap v6 y los análisis estadísticos se realizaron usando StataSE v12. Resultados: La muestra se compuso por 71 pacientes, siendo la mediana de edad de 50,1 años (Rango Intercuartil, RIC: 41,6-57,7) y un 45,1\% correspondió a mujeres. El 70,4\% afectó el hombro derecho. La evolución media de los síntomas fue de 5 meses (RIC: $2-24$ meses). Un 22,5\% recibió una o más infiltraciones previas y 47,9\% asistió a kinesioterapia. Las calcificaciones correspondieron principalmente al tipo 1 según la clasificación de Gartner y Heyer (73,3\%), con una mediana de tamaño de $14 \mathrm{~mm}$ en su eje mayor (RIC: 10-18 mm). Afectaron un tendón en $74,7 \%$, dos tendones en $18,3 \%$ y tres tendones en $7,0 \%$, siendo el tendón más frecuentemente afectado el supraespinoso (90,1\%). El 85,9\% presentó mejoría completa o significativa de los síntomas, 
requiriendo resolución quirúrgica con artroscopía tan sólo un 8,5\%. La mejoría final no tuvo asociación significativa con el tipo de Gartner y Heyer $(p=0,3)$, tamaño $(p=0,16)$, ni con el tiempo de evolución de los síntomas $(p=0,7)$. Conclusiones: Los pacientes con tendinitis cálcica que son sometidos a lavado y aspirado bajo ultrasonido tuvieron en su mayoría una resolución significativa o total de los síntomas a los dos meses de seguimiento, requiriendo artroscopia en un porcentaje menor. No se lograron identificar factores predictores de mala evolución de este procedimiento. Se requieren de más estudios para determinar sus ventajas por sobre el tratamiento médico.

Palabras clave: Tendinitis, tendinitis cálcica, manguito rotador, lavado por ultrasonido.

Guiloff S., et al. Tendinitis cálcica del manguito rotador y su lavado por ultrasonido. Rev Chil Radiol 2017; 23(3): 109115.Correspondencia: Esteban Hebel N. / ehebel@gmail.com

Trabajo enviado el 15 de marzo de 2017. Aceptado para publicar el 12 de septiembre de 2017.

\section{Introducción}

La tendinitis cálcica es una causa frecuente de dolor de hombro en el adulto, pudiendo llegar a resultar altamente incapacitante, afectando su independencia y calidad de vida. Se origina por depósito de cristales de hidroxiapatita en los tendones del manguito rotador con una prevalencia reportada del $2,5-7,5 \%$ de las radiografías en adultos asintomáticos ${ }^{1}$. Aproximadamente la mitad de éstos presentarán síntomas, siendo más frecuente en mujeres $(70 \%)$ y en mayores de 40 años $^{1,2}$. Una de las dificultades del manejo de la tendinitis cálcica es que, dado que es una entidad autolimitada, donde la calcificación se resuelve en forma espontánea, el tratamiento busca mejorar la calidad de vida y acelerar la recuperación a través del alivio sintomático, por lo que éste debe ser efectivo, sin complicaciones y mínimamente invasivo².

Tradicionalmente el tratamiento de primera elección es el manejo conservador, donde se incluye el uso de terapia física y un curso corto de antiinflamatorios no esteroidales ${ }^{3}$. Una aproximación alternativa es extraer la calcificación vía procedimientos guiados por ultrasonido. Estudios previos han mostrado resultados promisorios del lavado por ultrasonido de las calcificaciones como opción terapéutica, con múltiples técnicas descritas, sin embargo, ninguna de éstas han sido aplicadas en forma universal ${ }^{4-6}$. En caso de falla del manejo conservador y, como última instancia, se debe recurrir a la artroscopia.

El presente estudio tiene por objetivo describir la técnica de lavado y aspirado percutáneo de calcificaciones bajo ultrasonido en nuestro centro y evaluar sus resultados a corto plazo (dos meses).

\section{Material y métodos}

En este estudio aprobado por el comité de ética e investigación de nuestra institución, se recolectó retrospectivamente la información de todos los pacientes con diagnóstico de tendinitis cálcica sintomática de uno o más tendones del manguito rotador y que fueron sometidos a lavado y aspirado bajo ultrasonido de hombro entre los años 2011 y 2015 en Clínica Alemana de Santiago. Los datos incluyeron edad al momento del diagnóstico, tiempo de evolución de los síntomas, tratamientos previos efectuados, tendones comprometidos, lado afectado y uni o bilateralidad de la condición.

Previo al tratamiento, a todos los pacientes se les realizaron radiografías que incluyeron, al menos, dos planos del espacio (proyección anteroposterior y axial de escápula) y/o ecografía del hombro comprometido. Se reconocieron las calcificaciones del manguito rotador, su número, tamaño y localización, y con esta información se utilizó la clasificación morfológica según Gartner y Hayer ${ }^{7}$, que define las calcificaciones como de apariencia densa (tipo I), apariencia translúcida y nubosa (tipo III) y aquellas de morfología no clasificable (tipo II). El tamaño fue clasificado según Bosworth ${ }^{8}$, como pequeña (apenas visible), mediana $(<15 \mathrm{~mm})$ y grande $(>15 \mathrm{~mm})$.

Descripción de la técnica: El procedimiento se realiza con el paciente en decúbito supino y el brazo afectado en contacto con el cuerpo. El grado de rotación del hombro dependerá de la ubicación de la calcificación, intentando dejar el trayecto más corto y el mejor ángulo de abordaje entre la piel y la calcificación. Se realiza asepsia local y con técnica estéril (Figuras 1a y 1b) se anestesia con aguja $25 \mathrm{G}$ el trayecto de abordaje y la bursa subacromiosubdeltoídea (lidocaína 2\%) (Figura 1). Para nuestro estudio, se utilizó un transductor lineal multifrecuencial (12-5 ó 18-5 MHz) y equipos de ecografía Philips iU22 y Philips Epiq 7 para visualizar la calcificación y guiar el procedimiento (Figura 1c). A continuación, por el trayecto anestesiado se punciona la calcificación bajo guía ecográfica con trocar de 14G y en forma paralela se introduce una segunda aguja 19 ó 21G (Figura 1d). Se utilizan dos jeringas de $25 \mathrm{cc}$, una que realizará el aspirado a través de la aguja más gruesa y la otra introducirá suero fisiológico creando un sistema de lavado y aspirado. En caso de calcificaciones más extensas o múltiples, se puede realizar el lavado en más de un sector. En caso de calcificaciones tipo I de Gartner y Heyer se puede fragmentar con la aguja de mayor diámetro. El procedimiento se repite hasta que se ha logrado lavar la calcificación o el suero fisiológico se observe claro. Finalmente, se administra corticoides intrabursal (Cidoten rapilento ${ }^{\circledR}$ 
o Depomedrol ${ }^{\circledR} 40$ u 80 mg según la preferencia del médico tratante) (Figura 2). En caso de existir más de una calcificación, la técnica anterior se puede repetir para cada una de ellas. El tratamiento dura en promedio $20 \mathrm{~min}$. Inmediatamente después de finalizar el procedimiento, se prescribe un analgésico para usar en caso de ser necesario en los primeros días post-lavado. Todos los procedimientos fueron realizados por un radiólogo con más de 15 años de experiencia en ecografía de hombro e intervenciones guiadas bajo ultrasonido.

Todos los pacientes incluidos en el estudio tuvieron al menos una consulta con traumatólogo de seguimiento post-lavado con control aproximadamente a los dos meses, en donde se pesquisó si hubo mejoría de síntomas, evaluando específicamente el dolor y rango de movilidad del hombro afectado, clasificándolo posteriormente en mejoría completa en caso de ausencia de sintomatología, parcial en caso de que hubo mejoría de los síntomas, pero en forma incom-

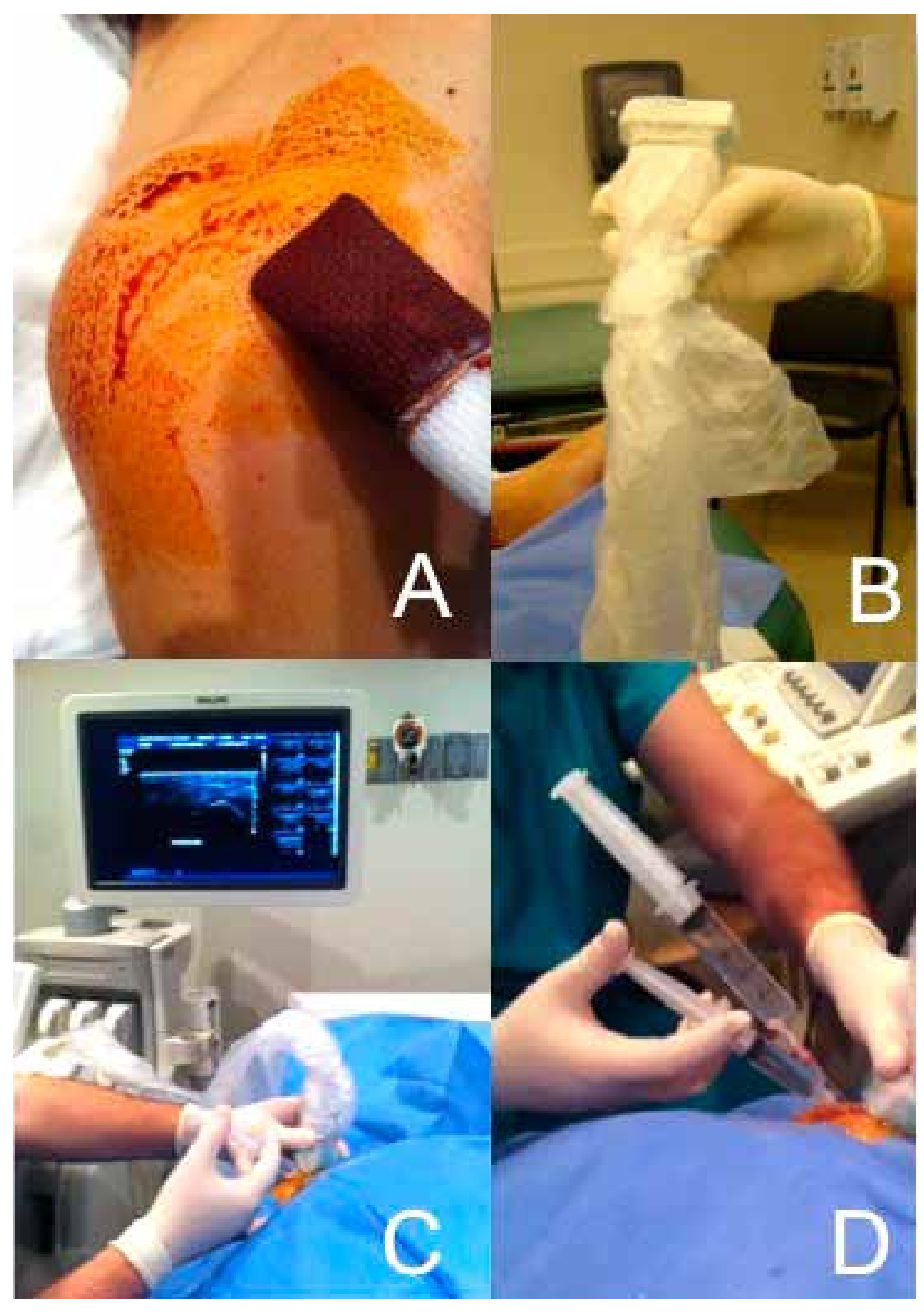

Figura 1. Procedimiento de punción y lavado bajo guía ecográfica. A y B. Demuestra el inicio del procedimiento bajo técnica aséptica. $C$. Se realiza la inyección del anestésico en el trayecto de abordaje. D. Se demuestra la técnica utilizando dos jeringas de 25 cc para la realización del lavado. 


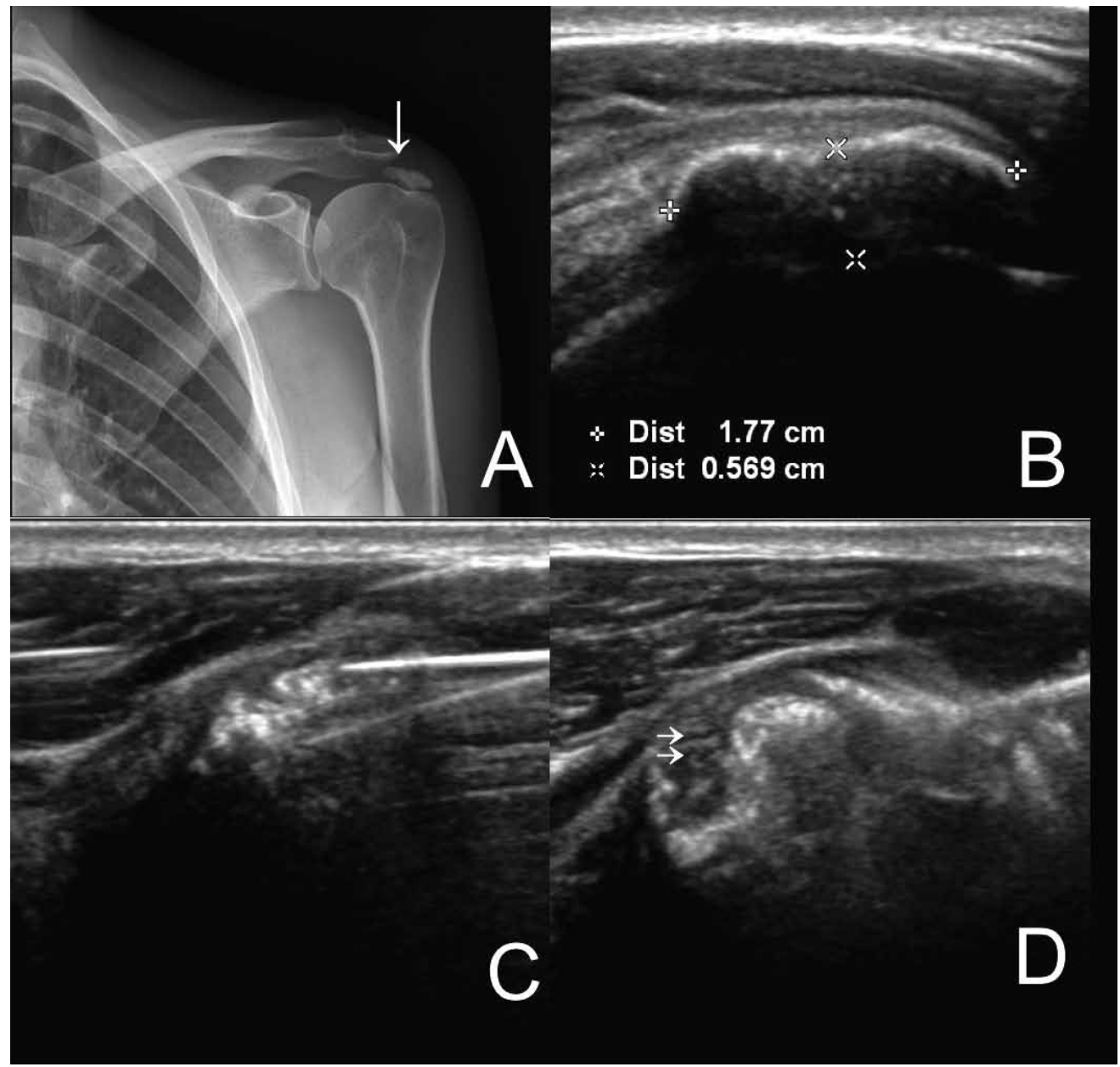

Figura 2. Mujer de 58 años con dolor de hombro izquierdo de 12 meses de evolución. A. Muestra calcificación densa, claramente definible (tipo I) de aproximadamente $20 \mathrm{~mm}$ en su eje mayor en la zona de inserción del tendón supraespinoso (flecha). B. Ecografía centrada en la inserción del músculo supraespinoso donde se identifica una gruesa calcificación, con sombra acústica posterior. C. Demuestra la utilización de dos agujas para el lavado de la calcificación. D. Distensión de la bursa posterior a la inyección de corticoides (doble flecha).

pleta o por último sin mejoría en los casos en que la sintomatología permaneció sin cambios significativos.

Se revisó la ficha clínica electrónica y todos los datos fueron recolectados y registrados en un formulario especialmente diseñado para este estudio, usando la plataforma RedCap 6.6.0 (Vanderbilt University, Nashville, TN).

Los análisis estadísticos univariados se realizaron usando medidas de tendencia central y dispersión para variables continuas. Los análisis bivariados se realizaron utilizando prueba de $\mathrm{X}^{2}$ para variables dicotómicas. Se consideraron intervalos de confianza del $95 \%$ y valores de significación estadística $p<0,05$. y todos los análisis estadísticos fueron realizados usando Stata Statistical Software versión 12 (StataCorp. 2011, College Station, TX).

\section{Resultados}

Se identificaron 94 pacientes, de los cuales 23 fueron excluidos por presentar datos incompletos. Los restantes 71 pacientes fueron incluidos en la presente revisión. La mediana de edad de los pacientes fue de 49,8 años (RIC: 41,8-56,8 años) (Figura 3) y 32 fueron mujeres $(45,1 \%)$. Los pacientes fueron tratados luego de una mediana de 5 meses con síntomas (RIC: 2-24 meses), 47,8\% había asistido a sesiones de kinesioterapia previa y previo al procedimiento $22,5 \%$ tuvo otra infiltración bursal con corticoides realizada sin guía por traumatólogo. La mayoría de pacientes presentó síntomas en el hombro derecho $(70,4 \%)$. Para este estudio, un $63,4 \%$ fue evaluado con ecografía de hombro y el $32,6 \%$ restante, con radiografía de hombro. La mayoría de los pacientes tuvo calcificaciones en un solo tendón con un $74,7 \%$, dos tendones en $18,3 \%$ y tres tendones en $7,0 \%$, siendo el más afectado el tendón del supraespinoso en un $90,1 \%$ (Figura 4). Las calcificaciones correspondieron principalmente al tipo II según la clasificación de Gartner y Heyer (73,3\%), con una mediana de tamaño de $14 \mathrm{~mm}$ en su eje mayor (RIC: $10-18 \mathrm{~mm}$ ) (Figura 5). En cuanto a la mejoría de los síntomas, 29 pacientes $(40,8 \%)$ presentaron mejoría completa de 
los síntomas y 32 pacientes (45,1\%) tuvieron mejoría parcial a los dos meses de seguimiento. Sólo 10 pacientes $(14,1 \%)$ no presentaron mejoría de síntomas con el procedimiento. Un $42,3 \%$ de los pacientes recibieron tratamiento adyuvante con antiinflamatorios no esteroidales y 53,4\% asistió a kinesioterapia. Sólo 11 pacientes $(15,5 \%)$ requirieron de un nuevo lavado y aspiración de microcalcificaciones y seis $(8,5 \%)$ terminaron en artroscopia de hombro para el manejo de esta patología. Al analizar la mejoría final respecto a las variables que pudieran haber incidido en el resultado, no se obtuvo asociación estadísticamente significativa con el tipo de Gartner y Heyer $(p=0,3)$, tamaño $(p=0,16)$, tanto calculado como variable continua o categórica, lado afectado $(p=0,5)$, ni el tiempo de evolución de los síntomas $(p=0,7)$ (Figura 6$)$.

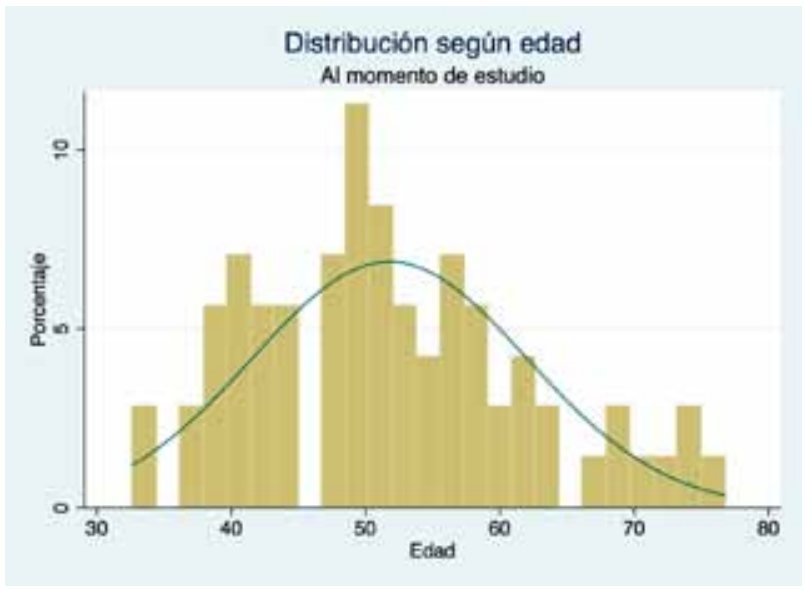

Figura 3. Histograma de distribución de edad de los pacientes al momento del procedimiento.

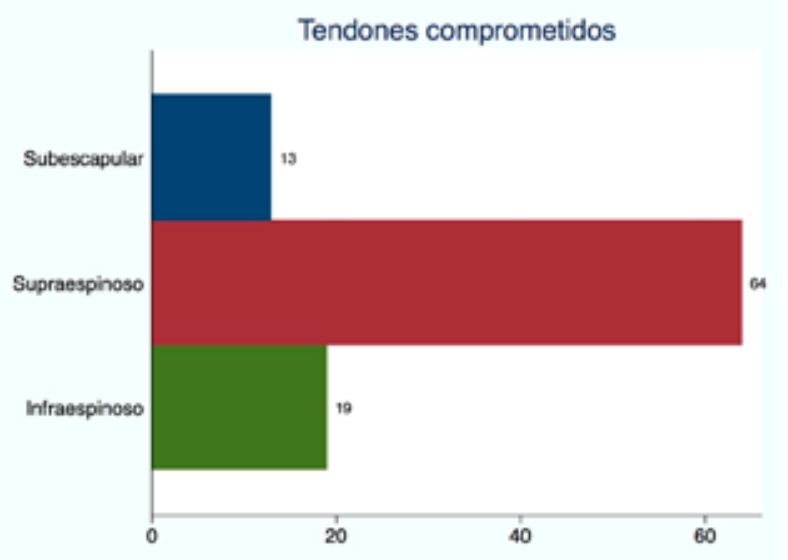

Figura 4. Distribución de la frecuencia de los tendones comprometidos en la muestra de pacientes analizados en nuestro estudio, demostrando que la gran mayoría de los pacientes presentaron afectación del tendón supraespinoso.

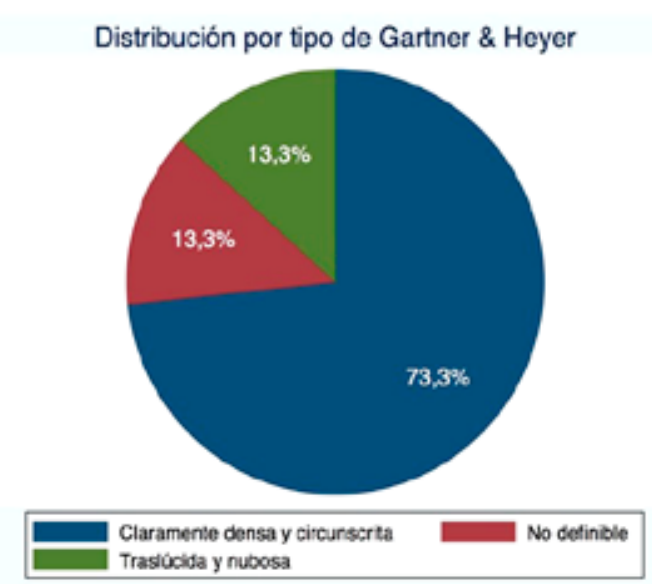

Distribución por tamaño de Bosworth

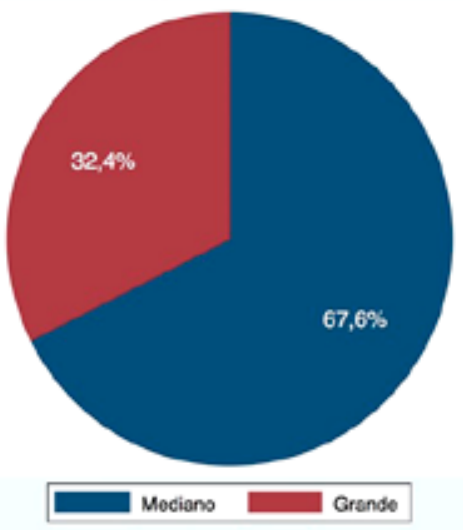

Figura 5. Caracterización de las calcificaciones por tipo radiológico según Gartner \&amp; Heyer y por tamaño según Bosworth, que demostró una frecuencia mayoritaria de calcificaciones "Claramente densas y circunscritas", así como aquellas clasificadas como medianas.

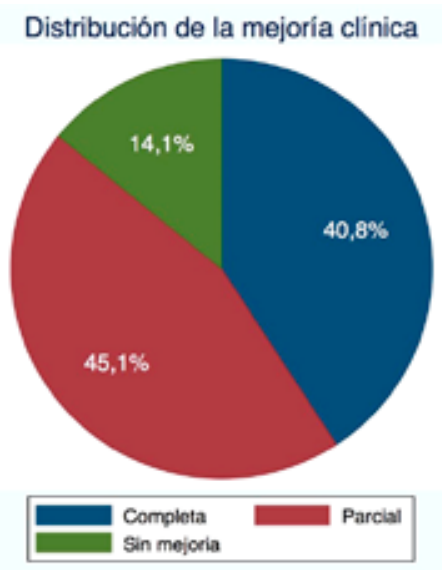

Figura 6. Distribución de los pacientes según su grado de mejoría clínica, destacando el que la gran mayoría tuvo mejoría total o parcial de sus síntomas, donde sólo un $14,1 \%$ de ellos no experimentó mejoría alguna con el tratamiento.

\section{Discusión}

Durante las últimas décadas, múltiples tratamientos han sido propuestos para el manejo conservador de la tendinitis cálcica del hombro. La iontoforesis con 
ácido acético y la terapia con ultrasonido no han probado ser más efectivos que la fisioterapia o el placebo $^{9,10}$. Las ondas de choque han demostrado su efectividad probando que, en el corto plazo, esta técnica resuelve las calcificaciones en $57-60 \%$ de los casos y logra una mejoría clínica sustancial o completa en 53-71\%. A largo plazo, las calcificaciones se resuelven en 47 a $93 \%$, y la mejoría clínica sustancial o completa se logra en $66-91 \%$. Sin embargo, es un procedimiento doloroso y requiere de equipamiento especial necesitando al menos tres sesiones para su aplicación separadas por 2-4 semanas ${ }^{11-13}$. La cirugía artroscópica es considerada una alternativa de segunda línea en caso de falla del tratamiento médico, siendo aún más efectiva que las ondas de choque a largo plazo, alcanzando una mejoría clínica sustancial o completa en $80-100 \%$ de los pacientes. Sin embargo, requiere siempre de hospitalización, rehabilitación y no está exenta de complicaciones ${ }^{13,14}$. El tratamiento percutáneo de la tendinitis cálcica no es un método nuevo; se realiza bajo apoyo fluoroscópico desde 1978, no obstante, su desventaja es el uso de radiación ionizante y la potencial dificultad de localizar el depósito cálcico ${ }^{15}$. El lavado y aspirado de calcificaciones bajo ultrasonografía fue descrito por primera vez en 1996 por Farin et $\mathrm{al}^{16}$. Se han descrito diversas variantes en la técnica entre los autores que han propuesto el uso de este procedimiento, siendo la principal diferencia el uso de una aguja ${ }^{6,17,18}$ versus dos agujas ${ }^{16,19}$. Nosotros elegimos usar dos agujas, una de 19-21G para introducir la solución salina y otra gruesa de 14G para aspirar el calcio. Consideramos, en nuestra experiencia, que usar agujas de diámetro más pequeño tienden a obstruirse durante la aspiración, mientras el uso de una de mayor diámetro aumenta el riesgo de dañar el tendón comprometido.

En nuestra institución, el procedimiento lo realizamos con el paciente en decúbito supino, ya que creemos que, en comparación con la posición sentada o semisentada, ésta es más confortable tanto para el paciente como para el operador. Algunos autores ${ }^{17,18}$ describen que la aspiración del calcio se debe realizar luego que la aguja ha fragmentado la calcificación. En nuestra experiencia y según los resultados reportados por otros estudios ${ }^{5,6}$, esta técnica causa frecuentemente que la aguja se obstruya, por lo que consideramos recomendable siempre inyectar la solución salina inicialmente.

Las posibles complicaciones reportadas derivadas del procedimiento mínimamente invasivo son poco frecuentes. Algunas de ellas son la presencia de reacciones vagales, hemorragia o hematoma local, infección y muy raramente síncope $\mathrm{e}^{4-6}$. Del cura et $\mathrm{al}^{6}$ pre medicó a más de la mitad de los pacientes de su estudio con un ansiolítico de rápida acción para prevenir pérdidas de conciencia y/o reacciones vagales. Creemos que no es necesario la pre medicación en forma extendida o rutinaria para estos pacientes, ya que la mala tolerancia al procedimiento es sólo anecdótica. En nuestro estudio no se presentaron complicaciones ni dolor durante el procedimiento sin pre medicación ni durante el mes de seguimiento.

Entre nuestros pacientes hubo mejoría clínica en el seguimiento a corto plazo, tanto del dolor como rango de movimiento, de manera completa o parcial en el $85,9 \%$ de los casos. Estos resultados son similares a los resultados más promisorios publicados para terapia con ondas de choque y no son peores en comparación a la artroscopia, pero a diferencia de éstas, el lavado y aspirado bajo visión ultrasonográfica es mínimamente invasivo, de menor duración, poco doloroso y de menor costo.

En cuanto a los factores predictores negativos descritos en la literatura, es decir que aumentan la probabilidad de una mala evolución clínica como lo son -por ejemplo- bilateralidad, calcificación localizada en relación a la porción anterior del acromion, extensión subacromial y depósito cálcico con un volumen mayor $\mathrm{a}>4500 \mathrm{~mm}^{3(20)}$, no tuvieron en nuestro estudio una asociación estadísticamente significativa con las distintas variables estudiadas, como el tiempo de evolución de síntomas, antecedente de tratamientos previos, ubicación de la(s) calcificación(es), tipo según clasificación de Gartner y Heyer ${ }^{7}$ y categorías de tamaño según Bosworth ${ }^{8}$ que condicionen una peor evolución clínica, al menos en el corto plazo.

Nuestro estudio tiene algunas limitaciones que deben tenerse en cuenta: Es un trabajo longitudinal retrospectivo, con un tiempo de seguimiento relativamente limitado. Pudiera ser de utilidad desarrollar un ensayo prospectivo controlado con mayor número de pacientes para identificar las variables que determinan el éxito del procedimiento en el mediano y largo plazo. Tampoco se controló con imágenes las calcificaciones en el seguimiento de los pacientes para comprobar si efectivamente la mejoría de la sintomatología se relaciona con la disminución de tamaño de éstas.

\section{Conclusiones}

Los pacientes con tendinitis cálcica del manguito rotador que son sometidos a lavado y aspirado bajo ultrasonido tienen en su mayoría una resolución significativa o total de los síntomas a los dos meses de seguimiento, requiriendo artroscopia en un porcentaje menor. No se lograron identificar factores predictores de mala evolución de este procedimiento, por lo que se puede proponer como alternativa válida para el manejo sintomático de cualquier paciente aquejado por esta condición, sin respuesta al tratamiento médico convencional. Se requieren de más estudios para determinar sus ventajas por sobre el tratamiento médico convencional y el impacto a largo plazo que tiene este tratamiento sobre la calidad de vida y recuperabilidad laboral de nuestros pacientes. 


\section{Referencias}

1. Speed CA, Hazleman BL. Calcific tendinitis of the shoulder. N Engl J Med 1999; 340: 1582-1584.

2. Hurt G., Baker C. Calcific tendinitis of the shoulder. Orthop Clin N Am 2003; 34: 567-563.

3. Kachewar SG., Kulkarni DS. Calcific tendinitis of the rotator cuff: A review. J Clin Diagn Res 2013; 7: 14821485.

4. Vignesh K., McDowall A., Simunovic N., Bhandari M., Choudur H. Efficacy of Ultrasound-Guided Percutaneous Needle Treatment of Calficific Tendinitis. AJR 2015; 204: 148-152.

5. Serafini G., Sconfienza L., Lacelli F., Silvestri E., Aliprandi A., Sardanelli F. Rotator Cuff Calcific Tendonitis: Short-term and 10-year Outcomes after Two-Needle US-guided Percutaneous Treatment- Nonrandomized Controlled Trial. Radiology 2009; 252: 157-164.

6. Del Cura J., Torre I., Zabala R., Legórburu A. Sonographically Guided Percutaneous Needle Lavage in Calcific Tendinitis of the Shoulder: Short- and LongTerm Results. AJR 2007; 189: 128-134.

7. Gartner J., Heyer A. Calcific tendinitis of the shoulder. Orthopade 1995; 24(3): 284-302.

8. Bosworth BM. Calcium deposits in the shoulder and subacromiali bursitis: A survey of 12,122 shoulder. JAMA 1941; 116: 2477-2482.

9. Ebenbichler G., Erdogmus CB., Resch KL., et al. UItrasound therapy for calcific tendinitis of the shoulder. N Engl J Med 1999; 340: 1533-1538.

10. Leduc BE., Caya J., Tremblay S., Bureau NJ. Treatment of calcific tendinitis of the shoulder by acetic acid iontophoresis: A double-blind randomized controlled trial. Arch Phys Med Rehabil 2003; 84: 1523-1527.

11. Gerdesmeyer L., Wagenpfeil S., Haake M., et al. Extracorporeal shockwave therapy for the treatment of chronic calcifying tendinitis of the rotator cuff: A randomized controlled trial. JAMA 2003; 290: 25732580.

12. Hsu CJ., Wang DY., Tseng KF., Fong YC., Hsu HC., Jim YF. Extracorporeal shock wave therapy for calcifying tendinitis of the shoulder. J Shoulder Elbow Surg 2008; 17(1): 55-59.

13. Rompe JD., Zoellner J., Nafe B. Shockwave therapy versus conventional surgery in the treatment of calcifying tendinitis of the shoulder. Clin Orthop Relat Res 2001; 387: 72-82.

14. Wittenberg RH., Rubenthaler F., Wolk Ludwig J., Willburger RE., Steffen R. Surgical or conservative treatment for chronic rotator cuff calcifying tendinitis: A matched-pair analysis of 100 patients. Arch Orthop Trauma Surg 2001; 121(1-2): 56-59.

15. Comfort TH., Arafiles RP. Barbotage of the shoulder with image-intensified fluoroscopic control of needle placement for calcific tendinitis. Clin Orthop Relat Res 1978; 135: 171-178.

16. Farin PU., Jaroma H., Soimakallio S. Rotator cuff calcifications: Treatment with US-guided technique. Radiology 1995; 195(3): 841-843.

17. Aina R., Cardinal E., Bureau NJ., Aubin B., Brassard P. Calcific shoulder tendinitis: Treatment with modified US-guided fine-needle technique. Radiology 2001; 221: 455-461.

18. Bradley M., Bhamra MS., Robson MJ. Ultrasoundguided aspiration of symptomatic supraspinatus calcific deposits. Br J Radiol 1995; 68: 716-719.

19. Galletti S., Magnani M., Rotini R., et al. The echoguided treatment of calcific tendinitis of the shoulder. Chir Organi Mov 2004; 89(4): 319-323.

20. Ogon P., Suedkamp NP., Jaeger M., Izadpanah K., Koestler W., Maier D. Prognostic factors in non operative therapy for chronic symptomatic calcific tendinitis of the shoulder. Arthritis Rheum 2009; 60: 2978-2984. 